PROCEEDINGS OF THE

AMERICAN MATHEMATICAL SOCIETY

Volume 127, Number 10, Pages 3049-3055

S 0002-9939(99)04878-9

Article electronically published on April 23, 1999

\title{
VOLUME, SURFACE AREA AND INWARD INJECTIVITY RADIUS
}

\author{
NOBUHIRO INNAMI
}

(Communicated by Christopher Croke)

\begin{abstract}
We show some relations among the volumes of domains in Euclidean spaces, their surface areas and the inward injectivity radii from their boundaries. In particular, we give an estimate for the upper bound of the ratios of their surface areas and volumes by means of inward injectivity radii. The upper bound seems to depend on their topological structures.
\end{abstract}

\section{INTRODUCTION}

Let $M$ be a compact connected oriented imbedded hypersurface in Euclidean $n$-space $E^{n}$ of class $C^{\infty}$ without boundary. Then, $M$ surrounds the compact domain $D(M)$ in $E^{n}$. Let $N$ be the inward unit normal vector field to $M$, and let $\varphi(p, t)=p+t N(p)$ for any $p \in M$ and $t \geq 0$. We call $r(p)=\sup \{r \mid d(\varphi(p, t), M)=$ $t$ for any $0 \leq t<r\}$ the inward injectivity radius of $M$ at $p$ and $r(M)=$ $\inf \{r(p) \mid p \in M\}$ the inward injectivity radius of $M$. Let Area $(M)$ and $\operatorname{Vol}(D(M))$ denote the $(n-1)$-dimensional volume of $M$ and the $n$-dimensional volume of $D(M)$, respectively. We say that $M$ is a tubular hypersurface with radius $a$ around a central set $C$ if $d(p, C)=a$ for any $p \in M$. The work in the present paper is motivated by the following theorem which is proved in [3].

Theorem 1. Suppose $n=3$. If $M$ is not a sphere topologically, then we have the inequality

$$
\frac{2}{r(M)} \geq \frac{\operatorname{Area}(M)}{\operatorname{Vol}(D(M))}
$$

and the equality sign holds if and only if $M$ is a tubular hypersurface with radius $r(M)$ around the central closed curve.

It is natural to ask whether the above theorem is true in the case of $n \geq 4$. The present paper attacks this problem. Let $\Sigma$ be the set of all hypersurfaces $M$ in $E^{n}$ as above and let $f: \Sigma \longrightarrow \mathbf{R}$ be the function given by

$$
f(M)=\frac{r(M)}{n} \frac{\operatorname{Area}(M)}{\operatorname{Vol}(D(M))}
$$

Received by the editors March 6, 1997 and, in revised form, December 23, 1997.

1991 Mathematics Subject Classification. Primary 53C20; Secondary 53A07, 53C42, 53C45.

Key words and phrases. Volume, surface area, injectivity radius, tube.

The author was partly supported by the Grants-in-Aid for Scientific Research, the Ministry of Education, Science and Culture, Japan. 
for any $M \in \Sigma$. Theorem 1 implies that $f$ for a tubular hypersurface in $E^{3}$ assumes a relative maximum $2 / 3$, since a small deformation of $M$ makes no change in the topological structure of $M$. In the same paper we saw that $f(M) \leq 1$ for any $M \in \Sigma$ and the equality sign holds if and only if $M$ is a round hypersphere in $E^{n}$. In this paper we investigate extremal points of $f$ on $\Sigma$. Here the terminology "extremal points" means that either relative maxima or minima are taken at those points. The conclusion of the paper is the following.

Conclusion 2. Suppose $M \in \Sigma$ is an extremal point of $f$ and is not a round hypersphere. Then,

$$
f(M) \leq \frac{n-1}{n} .
$$

The equality sign holds if and only if $M$ is a tubular hypersurface with radius $r(M)$ around a central closed curve.

The proof of Conclusion 2 consists of the following propositions.

Proposition 3. Suppose $M \in \Sigma$ is an extremal point of $f$ and the inward injectivity radius function $r$ is not constant in $M$. Then,

$$
f(M)<\frac{n-1}{n} .
$$

Proposition 4. Let $M \in \Sigma$. Suppose the inward injectivity radius function $r$ is constant in $M$. Then, $M$ is a tubular hypersurface in $E^{n}$ with radius $r(M)$ around a central $k$-dimensional submanifold $P$ in $E^{n}$.

Proposition 5. Suppose $M \in \Sigma$ is a tubular hypersurface in $E^{n}$ with radius $r(M)$ around a central $k$-dimensional submanifold $P$. If $k \geq 1$, then

$$
f(M) \leq \frac{n-1}{n},
$$

and the equality sign holds if and only if $k=1$.

Hotelling ([2]) and Weyl ([6]) have shown the equations of the volume and surface area of a tubular neighborhood of a submanifold $M$ in $E^{n}$ by means of intrinsic differential geometric quantities of $M$. Proposition 5 is not a direct consequence of their theorems if $k \geq 2$.

Proposition 3 proves Conclusion 2 when the inward injectivity radius function $r$ is not constant. Combining Proposition 5 with Proposition 4, Conclusion 2 is proved in the other case.

If $M \in \Sigma$ with $f(M)>(n-1) / n$ is not an extremal point, we deform $M$ without change of its topological structure so that $f(M)$ increases. Taking the limit, we have a topological hypersurface $M_{0}$. Conclusion 2 says that $M_{0}$ is a round hypersphere if $M_{0}$ is of class $C^{\infty}$. Then we know that $M$ is a hypersphere topologically and the higher dimensional case of Theorem 1 is true. The author has not yet succeeded in proving the differentiability of $M_{0}$.

The author would like to express his gratitude to Professor Kazuyuki Enomoto for showing him relevant references.

\section{Proof of Proposition 3}

Let $M \in \Sigma$ and let $g$ be a function of class $C^{\infty}$ on $M$. Let $M_{t}$ be a hypersurface in $\Sigma$ defined by the map $\varphi(p, t)=p+g(p) t N(p)$ where $p \in M$ and $t$ is a sufficiently 
small positive number. Then, we get

$$
\operatorname{Area}\left(M_{t}\right)=\int_{p \in M} \sqrt{\operatorname{det}\left(\left\langle E_{i}(p) \varphi, E_{j}(p) \varphi\right\rangle\right)} d M,
$$

where $\langle\cdot, \cdot\rangle$ is the natural inner product in $E^{n}, E_{i}(p)$ are the orthonormal eigenvectors of the second fundamental tensor $A$ of $M$ at $p, \operatorname{det}(\cdot)$ is the determinant of the matrix whose $(i, j)$-entries are $\left\langle E_{i}(p) \varphi, E_{j}(p) \varphi\right\rangle$, and $d M$ is the volume form of $M$ induced from the Riemannian metric of $M$,

$\operatorname{Vol}\left(D\left(M_{t}\right)\right)=\operatorname{Vol}(D(M))-\int_{p \in M} \int_{0}^{g(p) t}\left(-\kappa_{1}(p) t+1\right) \cdots\left(-\kappa_{n-1}(p) t+1\right) d t d M$,

where $\kappa_{i}(p)$ are the eigenvalues of $A$ at $p$ for $i=1, \cdots, n-1$. Differentiating them at $t=0$, we have

$$
\left.\left(\operatorname{Area}\left(M_{t}\right)\right)^{\prime}\right|_{t=0}=-(n-1) \int_{M} g H d M,
$$

where $H(p)=\left(\kappa_{1}(p)+\cdots+\kappa_{n-1}(p)\right) /(n-1)$ is the mean curvature of $M$ at $p$, and

$$
\left.\left(\operatorname{Vol}\left(D\left(M_{t}\right)\right)\right)^{\prime}\right|_{t=0}=-\int_{M} g d M
$$

If $r\left(M_{t}\right)$ is differentiable at $t=0$, then we have

$$
\begin{aligned}
\left.\left(f\left(M_{t}\right)\right)^{\prime}\right|_{t=0} & =\left.\frac{r\left(M_{t}\right)^{\prime}}{n}\right|_{t=0} \frac{\operatorname{Area}(M)}{\operatorname{Vol}(D(M))} \\
& -\frac{r(M)}{n} \frac{1}{\operatorname{Vol}(D(M))}\left(\int_{M} g\left((n-1) H-\frac{\operatorname{Area}(M)}{\operatorname{Vol}(D(M))}\right) d M\right) .
\end{aligned}
$$

Let $p_{0} \in M$ be a point in $M$ with $r\left(p_{0}\right)=\max \{r(p) \mid p \in M\}>r(M)$. We choose a function $h$ on $M$ such that $h(p)=1$ in a neighborhood $U$ of $p_{0}, h(p)=0$ in a neighborhood $V$ of the minimum set $\{p \in M \mid r(p)=r(M)\}$ of the inward injectivity radius function $r, h(p)>0$ for any $p \in M-V$ and $U \cap V=\emptyset$. Then, we set

$$
g(p)=h(p)\left((n-1) H(p)-\frac{\operatorname{Area}(M)}{\operatorname{Vol}(D(M))}\right)
$$

for any $p \in M$. It follows from Lemma 6 at the end of this section that $r\left(M_{t}\right)=$ $r(M)$ for any sufficiently small positive $t$, if $M_{t}$ is constructed by means of this function $g$. Thus, $\left.r\left(M_{t}\right)^{\prime}\right|_{t=0}=0$. Since $M$ is an extremal point of $f$, we have

$$
\int_{M} h\left((n-1) H-\frac{\operatorname{Area}(M)}{\operatorname{Vol}(D(M))}\right)^{2} d M=0 .
$$

It follows from this that

$$
\frac{\operatorname{Area}(M)}{\operatorname{Vol}(D(M))}=(n-1) H\left(p_{0}\right)<\frac{n-1}{r(M)},
$$

because all principal curvatures of $M$ at $p_{0}$ are less than $1 / r(M)$.

It remains to prove the following lemma which was applied in the above.

Lemma 6. If $M_{t}$ converges to $M$ as $t \rightarrow 0$ in the $C^{2}$-topology, then $r\left(q_{t}\right) \rightarrow r(p)$ for any sequence $q_{t} \in M_{t}$ converging to $p \in M$. 


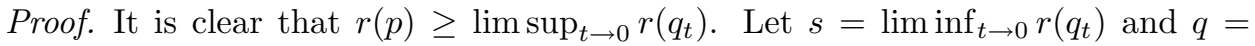
$p+s N(p)$. We have to prove that $q$ is a cut point of $M$ along the segment $T(p, q)$ from $p$ to $q$. Suppose for contradiction that $r(p)>s$. Then, $q$ is not a focal point to $M$ along $T(p, q)$. Moreover, $q_{t}+r\left(q_{t}\right) N\left(q_{t}\right)$ are not focal points to $M_{t}$ for all sufficiently small positive $t$, because $M_{t}$ converges in the $C^{2}$-topology. This implies that there exist at least two minimizing segments from $q$ to $M$. However, the existence of two minimizing geodesics contradicts that $r(p)>s$. This completes the proof.

\section{Proof of Proposition 4}

Let $\varphi_{t}: M \longrightarrow E^{n}$ be a map given by $\varphi_{t}(p)=p+t N(p)$ for any $p \in M$ and let $P=\varphi_{r(M)}(M)$. It is obvious that $P$ has no interior point as a subset in $E^{n}$. We first notice that $D(M)$ is the $r(M)$-tubular neighborhood around $\mathrm{P}$ and $M$ is the $r(M)$-tubular hypersurface around $P$, that is, $M=\left\{p \in E^{n} \mid d(p, P)=r(M)\right\}$. It remains to prove that $P$ is a smooth submanifold without boundary. It should be remarked that the boundary of $P$ may be non-empty if $M$ is not of class $C^{2}$. However, it is not the case in the present situation.

Let $\Delta(p)=\left\{X_{p} \in T_{p} M \mid d \varphi_{r(M)}\left(X_{p}\right)=0\right\}$ and $\mu(p)=\operatorname{dim} \Delta(p)$ for any $p \in$ $M$. Then, $\mu$ is upper semi-continuous, that is, $\lim _{\sup _{q \rightarrow p}} \mu(q) \leq \mu(p)$. Let $\ell=$ $\min \{\mu(p) \mid p \in M\}$ and $U=\{p \in M \mid \mu(p)=\ell\}$. By virtue of the upper semicontinuity, the set $U$ is open in $M$. We prove in Lemmas 7 through 14 that $U$ is closed, and at the same time that $P$ is a smooth submanifold without boundary.

Lemma 7. Let $p$ be a point in $U$ and $X_{p} \in T_{p} M$. The following are equivalent.

$$
\begin{gathered}
X_{p} \in \Delta(p) . \\
A_{p}\left(X_{p}\right)=\frac{1}{r(M)} X_{p} . \\
d \varphi_{t}\left(X_{p}\right)=\left(-\frac{t}{r(M)}+1\right) X_{p} \quad \text { for } \quad 0 \leq t \leq r(M) .
\end{gathered}
$$

Proof. In general,

$$
d \varphi_{t}\left(X_{p}\right)=-t A_{p}\left(X_{p}\right)+X_{p}
$$

holds for any tangent vector $X_{p} \in T_{p} M$ and $t \geq 0$. Lemma 7 is a direct consequence of this fact.

If $\ell=n-1$, then $U=M$, and, hence, $M$ is a round hypersphere in $E^{n}$. From now on, we assume that $\ell<n-1$.

Lemma 8. $\Delta$ is an involutive $\ell$-dimensional smooth distribution on $U$.

Proof. Let $X, Y \in \Delta$. Recall that $\varphi_{t}: U \longrightarrow \varphi_{t} U$ is a diffeomorphism for $0 \leq t<$ $r(M)$. Then, we see that

$$
d \varphi_{t}\left([X, Y]_{p}\right)=\left(-\frac{t}{r(M)}+1\right)^{2}[X, Y]_{\varphi_{t}(p)}
$$

for any $0 \leq t<r(M)$. Therefore, we get $d \varphi_{r(M)}\left([X, Y]_{p}\right)=0$ as $t \longrightarrow r(M)$. This implies that $[X, Y]_{p} \in \Delta(p)$, and, hence, $\Delta$ is involutive (see [5]). This completes the proof. 
Let $W(p)$ be the maximal integral manifold of $\Delta$ in $U$ through $p \in U$. Let $S^{n-1}\left(p_{0}, a\right)$ denote the hypersphere with center $p_{0}$ and radius $a$.

Lemma 9. $W(p) \subset S^{n-1}\left(p_{0}, r(M)\right)$, where $p_{0}=p+r(M) N(p)$.

Proof. Let $c(q)=q+r(M) N(q)$ for $q \in W(p)$. Then, for any $X_{q} \in \Delta(q)=T_{q} W(p)$, we see that

$$
X_{q} c=X_{q}+r(M)\left(-A_{q}\left(X_{q}\right)\right)=0 .
$$

Therefore, we get

$$
q+r(M) N(q)=p_{0},
$$

where $p_{0}=\varphi_{r(M)}(p)$. This implies that $\left\|q-p_{0}\right\|^{2}=r(M)^{2}$ for any $q \in W(p)$. This completes the proof.

Lemma 10. $W(p)$ is totally geodesic in both $S^{n-1}\left(p_{0}, r(M)\right)$ and $M$.

Proof. Let $S(q)$ be the linear subspace spanned by $N(q)$ at $q \in W(p)$. We have to show that $\left(\nabla_{X} Y\right)_{q} \in \Delta(q) \oplus S(q)$ for any $X, Y \in \Delta$ and $q \in W(p)$ where $\nabla$ denotes the Levi-Civita connection in $M$. To show this it is enough to see that $\Delta(q) \oplus S(q)=\Delta(p) \oplus S(p)$ for any $q \in W(p)$ because they are linear subspaces. Let $\Gamma(q)$ be the orthogonal complement of $\Delta(q)$ in $T_{q} M$. It should be noted that $d \varphi_{r(M)} \Gamma(q)=\Gamma(q)$ for any $q \in W(p)$. We shall prove that $\Delta(q) \oplus S(q)$ is orthogonal to $\Gamma(p)$ for any $q \in W(p)$. Notice that it is true if $\langle N(q), \Gamma(p)\rangle=0$ for any $q \in W(p)$, since

$$
0=X_{q}\langle N(q), v\rangle=\left\langle-\frac{1}{r(M)} X_{q}, v\right\rangle
$$

for any $X_{q} \in \Delta(q)=T_{q} W(p)$ and $v \in \Gamma(p)$, and, hence, $\Delta(q) \perp \Gamma(p)$. Suppose for contradiction that there is a vector $v \in \Gamma(p)$ such that $\left\langle d \varphi_{r(M)}(v), N(q)\right\rangle<0$. Let $c:[0, \varepsilon) \longrightarrow M$ be a curve with $\dot{c}(0)=v$ and let $b(s)=c(s)+r(M) N(c(s))$. Then, $b(0)=p_{0}, \dot{b}(0)=d \varphi_{r(M)}(v)$ and $b(s) \in P$ for any $s \in[0, \varepsilon)$. Since $\langle\dot{b}(0), N(q)\rangle<0$, there is an $s \in[0, \varepsilon)$ such that $d(b(s), q)<d\left(p_{0}, q\right)=r(M)$, contradicting the assumption that $M$ is the $r(M)$-tubular hypersurface around $P$. This completes the proof.

As a consequence of Lemma 10, we see that $\Gamma(q)=\Gamma(p)$ for $q \in W(p)$. Moreover, $\Gamma(q)=\Gamma(p)$ if $\varphi_{r(M)}(p)=\varphi_{r(M)}(q)$ for any $p, q \in U$.

Lemma 11. Let $\bar{P}=\varphi_{r(M)}(U)$. Then, $\bar{P}$ is a smooth submanifold with dimension $k=n-\ell-1$.

Proof. Let $p_{0} \in \bar{P}$. We can find a point $p \in U$ with $\varphi_{r(M)}(p)=p_{0}$. It follows from Lemma 8 that there is a cubic coordinate neighborhood $\left(V ; x^{1}, \cdots, x^{n-1}\right)$ of $p$ such that $x^{1}(p)=0, \cdots, x^{n-1}(p)=0$, and $W(q) \cap V$ is a slice defined by $x^{\ell+1}=a_{1}, \cdots, x^{n-1}=a_{k}$ for any $q \in V$ where $a_{1}, \cdots, a_{k}$ are some constants (cf. $[5])$. Let $V(p)=\left\{q \in V \mid x^{1}(q)=0, \cdots, x^{\ell}(q)=0\right\}$ and let $\bar{V}\left(p_{0}\right)=\varphi_{r(M)}(V(p))$. By construction of $\bar{V}\left(p_{0}\right)$, we see that $\psi=\varphi_{r(M)} \mid V(p)$ is a diffeomorphism from $V(p)$ onto $\bar{V}\left(p_{0}\right)$, and, therefore, $\left(\bar{V}\left(p_{0}\right) ; x^{\ell+1} \circ \psi^{-1}, \cdots, x^{n-1} \circ \psi^{-1}\right)$ is a coordinate neighborhood of $p_{0}$ in $\bar{P}$. This completes the proof. 
We have seen that $W(p)$ is a connected component of $U \cap S^{\ell}\left(p_{0}, r(M)\right)$, where

$$
S^{\ell}\left(p_{0}, r(M)\right)=S^{n-1}\left(p_{0}, r(M)\right) \cap\left(p_{0}+\Delta(p) \oplus S(p)\right) .
$$

Suppose $\ell=0$. Then, $W(p)=p$ and $\bar{P}$ has no boundary, because $\operatorname{dim} \bar{P}=n-1$ and $M$ is of class $C^{\infty}$. This implies that $M$ is not connected, contradicting the assumption that $M$ is connected. Therefore, $\ell \geq 1$.

Lemma 12. $W(p)=S^{\ell}\left(p_{0}, r(M)\right)$ for any $p \in U$.

Proof. Suppose $W(p) \neq S^{\ell}\left(p_{0}, r(M)\right)$. Then, we can find a boundary point $q \in$ $\partial W(p)$ in $S^{\ell}\left(p_{0}, r(M)\right)$. It is clear that $q \in \partial U$. Let $q_{m} \in W(p)$ with $q_{m} \rightarrow q$. Notice that if the principal curvatures $\tau_{m}$ of $M$ at $q_{m}$ are different from $1 / r(M)$, then they are written as

$$
\tau_{m}=\frac{-\kappa_{m}}{-r(M) \kappa_{m}+1}
$$

where $\kappa_{m}$ are principal curvatures of $\bar{V}\left(p_{0}\right)$ at $p_{0}$ with respect to $-N\left(q_{m}\right)$. The principal curvatures of $\bar{V}\left(p_{0}\right)$ at $p_{0}$ are bounded, that is, there is a positive constant $K$ such that $\left|\kappa_{m}\right|<K$, since $\bar{V}\left(p_{0}\right)$ is smooth at $p_{0}$. This shows that $\tau_{m}$ cannot be close to $1 / r(M)$, and, hence, $\operatorname{dim} \Delta(q)=\ell$. This contradicts that $q \in \partial U$. This completes the proof.

It follows from the definition of $r(M)$ that the maximal principal curvature is $1 / r(M)$ on $U$. The following lemma gives us an estimate for the others.

Lemma 13. Let $q \in U$. If a principal curvature $\kappa$ at $q$ is not $1 / r(M)$, then $\kappa \leq$ $1 /(2 r(M))$.

Proof. Suppose for contradiction that there exist a point $q \in U$ and a principal curvature $\kappa$ of $M$ at $q$ with $1 / r(M)>\kappa>1 /(2 r(M))$. Let $q_{1}$ be the antipodal point of $q$ in $S^{\ell}\left(q_{2}, r(M)\right)$ where $q_{2}=\varphi_{r(M)}(q)$. Since $2 r(M)>1 / \kappa>r(M)$, there exists a focal point $q_{3}$ of $q$ to $M$ in the segment $T\left(q_{2}, q_{1}\right)$ joining $q_{2}$ to $q_{1}$. It should be noted that $q_{3}$ is also a focal point to $\bar{P}$ along the segment $T\left(q_{2}, q_{3}\right)$. This implies that the segment $T\left(q_{1}, q_{2}\right)$ is not a shortest join from $q_{1}$ to $P$ (see [4]), contradicting that $M$ is the $r(M)$-tubular hypersurface around $P$. This completes the proof.

Lemma 14. $U$ is closed and $P$ is a smooth submanifold without boundary.

Proof. This is clear from Lemma 13 and Lemma 11.

\section{Proof of Proposition 5}

If $\operatorname{dim} P=k=1$, then the equation $f(M)=(n-1) / n$ is a simple application of a theorem of Hotelling ([1], [2]). Here we assume that $k \geq 2$. Let $\nu(P)$ be the unit normal vector bundle of $P$ and $\lambda_{1}(v), \cdots, \lambda_{k}(v)$ the principal curvatures of $P$ with respect to $v \in \nu(P)$. Put $r=r(M)$. Recall (cf. [1])

$$
\begin{gathered}
\operatorname{Vol}(D(M))=\int_{0}^{r} \int_{\nu(P)}\left(1-t \lambda_{1}\right) \cdots\left(1-t \lambda_{k}\right) t^{n-k-1} d P d \Theta d t, \\
\operatorname{Area}(M)=\int_{\nu(P)}\left(1-r \lambda_{1}\right) \cdots\left(1-r \lambda_{k}\right) r^{n-k-1} d P d \Theta
\end{gathered}
$$


where $d P$ and $d \Theta$ are the volume forms of $P$ and the unit sphere $S^{n-k-1}$, respectively. Put

$$
F(t)=\left(1-t \lambda_{1}\right) \cdots\left(1-t \lambda_{k}\right) t^{n-k-1}
$$

for $0 \leq t<r$. Since we assume that $M$ is an imbedded hypersurface, it follows that $\lambda_{i}<1 / t$ for $0<t \leq r$, and, hence, $t \lambda_{i}<1$. Moreover, we see that

$$
-1<t \lambda_{i}<1
$$

for $0 \leq t<r$, since $\lambda_{i}(v)=-\lambda_{i}(-v)$ for any $v \in \nu(P)$. Differentiating $F$ with respect to $t$, we get

$$
F^{\prime}(t)=\left(\sum_{i=1}^{k} \frac{-t \lambda_{i}}{1-t \lambda_{i}}+(n-k-1)\right) \frac{F(t)}{t} .
$$

By (7), we get

$$
\frac{-t \lambda_{i}}{1-t \lambda_{i}}<\frac{1}{2}
$$

for $0<t<r$. Substituting (9) into (8), we have

$$
t F^{\prime}(t)<(n-2) F(t)
$$

for $0<t<r$, since $k / 2 \leq k-1$. Integrating (10) on the interval [0,r], we get

$$
r F(r)<(n-1) \int_{0}^{r} F(t) d t .
$$

Again, integrating (11) over $\nu(P)$, we have

$$
r \operatorname{Area}(M)<(n-1) \operatorname{Vol}(D(M)) \text {. }
$$

This completes the proof of Proposition 5.

\section{REFERENCES}

1. A. Gray, Tubes, Reading Mass., Addison-Wesley, 1990. MR 92d:53002

2. H. Hotelling, Tubes and spheres in n-spaces, and a class of statistic problems, Amer. J. Math. 61 (1939), 440-460.

3. N. Innami, Isoperimetric inequalities depending on injectivity radius from boundary, Complex Structures And Vector Fields, (Editors S. Dimiev \& K. Sekigawa), World Scientific, Singapore, 1995, pp. 36-45. MR 96m:53062

4. H. Nakagawa, Global Riemannian Geometry, Kaigaishuppanboueki, Tokyo, 1977, (in Japanese).

5. F. Warner, Foundations of Differentiable Manifolds And Lie Groups, Scott, Foresman and Company, Illinois, 1971. MR 45:4312

6. H. Weyl, On the volume of tubes, Amer. J. Math. 61 (1939), 461-472.

Department of Mathematics and Information, Graduate School of Science and TechNOLOGy, NiIgata University, NiIgata, 950-2181, JAPAN

E-mail address: innami@math.sc.niigata-u.ac.jp 